\title{
Quark number susceptibility around the critical end point
}

\author{
Min $\mathrm{He}^{a}$, Jian-Feng $\mathrm{Li}^{a}$, Wei-Min Sun ${ }^{a, b}$, and Hong-Shi Zong ${ }^{a, b}$ \\ a Department of Physics, Nanjing University, Nanjing 210093, P. R. China and \\ b Joint Center for Particle, Nuclear Physics and Cosmology, Nanjing 210093, China
}

\begin{abstract}
The quark number susceptibility is expressed as an integral in terms of dressed quark propagator and dressed vector vertex. It is then investigated with the latter two- and three-point functions confronted with a Dyson-Schwinger equation model which accommodates both finite temperature and baryon chemical potential. The critical end point in the phase diagram is identified and the behavior of the quark number susceptibility around the critical end point is highlighted. The qualitative features found agree with recent lattice QCD simulation results.
\end{abstract}

Key-words: critical end point, quark number susceptibility, Dyson-Schwinger equation

E-mail: zonghs@chenwang.nju.edu.cn.

PACS Numbers: 11.10.Kk, 11.15.Tk, 11.30.Qc 


\section{INTRODUCTION}

Underlying our understanding of strong interaction, Quantum Chromodynamics (QCD) is remarkable in that its fundamental degrees of freedom - quarks and gluons are confined and the (approximate) chiral symmetry in the light quark sector is dynamically broken. On the other hand, at sufficiently high temperatures and/or baryon chemical potentials, QCD is believed to undergo a phase transition into the deconfined quark gluon plasma (QGP) (for a recent review, see, e.g., [1]), and probably at the same time, the spontaneously broken chiral symmetry gets restored [2]. It is the main task of the on-going BNL-RHIC and one of the goals of CERN-LHC to create and probe such a new state of QCD matter.

The phase transition of confined hadronic matter to QGP at finite temperature and vanishing baryon chemical potential has been extensively studied by lattice QCD simulations as well as phenomenological models. The chiral phase transition of QCD with two flavors in the chiral limit is likely to be of second order [3] and turns to a smooth crossover in the case of physical finite current quark masses [4, 5, 6]. At finite chemical potentials and lower temperatures, more and more robust lattice simulations indicate that QCD experiences a first-order phase transition [7, 8]. Such a scenario of QCD phase transition suggests that there should exist a critical end point (CEP) on the phase diagram - the point where the first-order phase transition line terminates (for a recent review on CEP, and especially the argument that CEP must exist, see [9]).

The search for the QCD CEP has attracted considerable attention over the years from both theoretical and experimental aspects [10, 11, 12, 13, 14, 15, 16]. Note that the end point of a first-order phase transition line is a critical point of the second order - in this connection, the water liquid-vapor phase transition makes a more familiar example. This means that the CEP would be characterized by enhanced long wave-length fluctuations which lead to singularity in thermodynamic observables. The authors of Ref. [10] suggest that quark number susceptibility should develop some singularity near the CEP and its divergence could be a signal of the CEP. From then on, a lot of phenomenological model investigations [17, 18, 19, 20, 21, 22] as well as lattice simulations [23, 24, 25] toward this susceptibility have been attempted, all aimed at determining the location of the CEP on the temperature $(T)$-chemical potential $(\mu)$ plane. On the experimental side, recent plans for verification of the CEP in heavy ion collisions have concentrated on energy scans to access 
as broad as possible range of $T$ and $\mu$ values necessary for the search for the CEP. It is argued that the range of $T$ and $\mu$ values for the CEP search can be accessible by combined results from energy scans at FAIR, SPS and RHIC [16]. Also notable is that, apart from susceptibilities, transport coefficients, like the ratio of viscosity to entropy density $(\eta / s)$ can be other probes for the CEP [15, 16, 26].

In the present work, we extend our previous continuum study of quark number susceptibility [3] to finite chemical potential in a Dyson-Schwinger equation model and aim at locating the CEP. We first derive a closed integral expression for the susceptibility in terms of QCD dressed quark propagator and dressed vector vertex. This integral formula proves to reproduce the exact analytical result in the case of a free quark gas. In the next section, with a model gluon propagator input, the 2- and 3-point functions are confronted with Dyson-Schwinger equation (DSE) and Bethe-Salpeter equation (BSE). With these results, the quark number susceptibility is then calculated and the characteristic behavior of the susceptibility near the CEP is highlighted and its implications analyzed. The last section is devoted to some concluding remarks. Throughout this work, we work with Euclidean space metric: $\left\{\gamma_{\mu}, \gamma_{\nu}\right\}=2 \delta_{\mu \nu}$.

\section{ANALYTICAL RESULTS}

\section{A. Derivation of a closed integral expression for the quark number susceptibility}

The quark number susceptibility is defined as the derivative of quark number density with respect to the baryon chemical potential

$$
\chi=\frac{\partial n}{\partial \mu} .
$$

To start our derivation, we write the quark number density as

$$
n=<\psi^{+} \psi>=<\bar{\psi}_{i}\left(\gamma_{4}\right)_{i j} \psi_{j}>=(-) N_{c} N_{f} \int \frac{d^{4} p}{(2 \pi)^{4}} \operatorname{tr}_{\gamma}\left[G(p, \mu) \gamma_{4}\right]
$$

where $G$ denotes the quark propagator, $N_{c}, N_{f}$ the color factor and the number of flavors respectively, and the trace is taken over Dirac indices. Here, the minus sign comes from the anti-commutation property of the fermion fields.

Substituting Eq. (2) into Eq. (1) and adopting the identity

$$
\frac{\partial G(p, \mu)}{\partial \mu}=(-) G(p, \mu) \frac{\partial G^{-1}(p, \mu)}{\partial \mu} G(p, \mu),
$$


we have

$$
\chi=N_{c} N_{f} \int \frac{d^{4} p}{(2 \pi)^{4}} \operatorname{tr}_{\gamma}\left[G(p, \mu) \frac{\partial G^{-1}(p, \mu)}{\partial \mu} G(p, \mu) \gamma_{4}\right] .
$$

Recall that the well-known Ward identity reads

$$
i \Gamma_{\mu}(p, 0)=\frac{\partial G^{-1}(p)}{\partial p_{\mu}}
$$

where $p$ denotes the relative momentum of the vector vertex and the corresponding total momentum vanishes. Note that at finite temperature and chemical potential, the fourth component of momentum $p_{4}=\omega_{n}+i \mu$, with $\omega_{n}$ being fermion Matsubara frequencies. Then

$$
(-) \Gamma_{4}(p, 0 ; \mu)=\frac{\partial G^{-1}(p, \mu)}{\partial \mu} \text {. }
$$

Putting this equation into Eq. (4) and replacing the integration over the fourth component of momentum with explicit summation over Matsubara frequencies, we get the quark number susceptibility at finite temperature and chemical potential in the imaginary-time formalism

$$
\chi(T, \mu)=(-) N_{c} N_{f} T \sum_{n=-\infty}^{+\infty} \int \frac{d^{3} p}{(2 \pi)^{3}} \operatorname{tr}_{\gamma}\left[G\left(\widetilde{p}_{n}\right) \Gamma_{4}\left(\widetilde{p}_{n}, 0\right) G\left(\widetilde{p}_{n}\right) \gamma_{4}\right],
$$

where $\widetilde{p}_{n}=\left(\vec{p}, \omega_{n}+i \mu\right)$ with fermion frequencies $\omega_{n}=(2 n+1) \pi T$.

Thus a model-independent closed integral formula is obtained, which expresses the quark number susceptibility in terms of the dressed quark propagator and the dressed vector vertex, both of the latter objects being basic quantities in quantum field theory. The DSE-BSE approach provides an ideal framework to confront these quantities non-perturbatively and then calculate the quark number susceptibility.

\section{B. The free quark number susceptibility}

Before going into the model calculation of the quark number susceptibility, let us apply the foregoing analytical expression for the susceptibility to the case of a free quark gas. In this case, the dressed vector vertex reduces to the bare one

$$
\Gamma_{4}\left(\widetilde{p}_{n}, 0\right) \longrightarrow \gamma_{4}
$$

and the free quark propagator reads

$$
G^{\text {free }}\left(\widetilde{p}_{n}\right)=\frac{1}{i \gamma \cdot \widetilde{p}_{n}+m}
$$


with $m$ the degenerated light quark current mass. Substituting Eqs. (8) and (9) into Eq. (7) and evaluating the trace, one gets

$$
\chi^{\text {free }}(T, \mu)=4 N_{c} N_{f} T \sum_{n=-\infty}^{+\infty} \int \frac{d^{3} p}{(2 \pi)^{3}} \frac{\left(\omega_{n}+i \mu\right)^{2}-E_{\vec{p}}^{2}}{\left[\left(\omega_{n}+i \mu\right)^{2}+E_{\vec{p}}^{2}\right]^{2}},
$$

where $E_{\vec{p}}=\sqrt{\vec{p}^{2}+m^{2}}$. With the aid of the identity

$$
\sum_{n=-\infty}^{+\infty} \frac{1}{i \omega_{n}-x}=\frac{\beta}{2} \times \frac{1-e^{\beta x}}{1+e^{\beta x}}
$$

one immediately arrives at

$$
\chi^{\text {free }}(T, \mu)=2 N_{c} N_{f} \beta \int \frac{d^{3} p}{(2 \pi)^{3}}\left\{\frac{e^{\beta\left(E_{\vec{p}}-\mu\right)}}{\left[e^{\beta\left(E_{\vec{p}}-\mu\right)}+1\right]^{2}}+\frac{e^{\beta\left(E_{\vec{p}}+\mu\right)}}{\left[e^{\beta\left(E_{\vec{p}}+\mu\right)}+1\right]^{2}}\right\} .
$$

This equation can be rewritten as

$$
\chi^{\text {free }}(T, \mu)=2 N_{c} N_{f} \frac{\partial}{\partial \mu} \int \frac{d^{3} p}{(2 \pi)^{3}}\left\{\frac{1}{e^{\beta\left(E_{\vec{p}}-\mu\right)}+1}-\frac{1}{e^{\beta\left(E_{\vec{p}}+\mu\right)}+1}\right\} .
$$

This integral is nothing but the free quark gas density from the Fermi-Dirac statistics. Eq. (13) is just a re-expression of the definition of the quark number susceptibility (Eq.(1)) for a free quark gas. The integration of Eq. (13) can be analytically solved in the chiral limit $(m=0)$

$$
\chi^{\text {free }}(T, \mu)=N_{f}\left(T^{2}+\frac{3 \mu^{2}}{\pi^{2}}\right)
$$

We see that the integral formula Eq. (7) for the quark number susceptibility reproduces exactly the Fermi-Dirac statistics result for a free quark gas. This is to be expected in advance and can be regarded as a consistent check of Eq. (7).

\section{MODEL CALCULATION}

\section{A. The dressed quark propagator}

In order to calculate the quark number susceptibility, we have to evaluate first the dressed quark propagator and dressed vector vertex at finite temperature and chemical potential. This will be conducted systematically and consistently in the rainbow-DSE and ladder-BSE [27] framework with a separable effective interaction model.

The DSE-BSE provides a non-perturbative continuum approach for the exploration of strong interaction physics. Its practical form, namely the rainbow-ladder truncation, has 
given us fascinating insights into the remarkable dynamical chiral symmetry breaking and confinement [28, 29] and found successful applications in describing light mesons in the pseudo-scalar and vector channels (for review articles, see [30]). The rainbow DSE for the quark propagator reads

$$
G(p)^{-1}=i \gamma \cdot p+m+\frac{4}{3} \int \frac{d^{4} p}{(2 \pi)^{4}} g^{2} D_{\mu \nu}^{e f f}(p-q) \gamma_{\mu} G(q) \gamma_{\nu}
$$

and the ladder inhomogeneous BSE for the dressed vector vertex reads

$$
\Gamma_{\mu}(p, P)=\gamma_{\mu}-\frac{4}{3} \int \frac{d^{4} q}{(2 \pi)^{4}} g^{2} D_{\rho \sigma}^{e f f}(p-q) \gamma_{\rho} G\left(q_{+}\right) \Gamma_{\mu}(q, P) G\left(q_{-}\right) \gamma_{\sigma},
$$

where $P$ denotes the total momentum, $q_{ \pm}=q \pm P / 2$ and $D_{\mu \nu}^{e f f}(p-q)$ the effective gluon propagator, which is usually a phenomenological input in practice (There are also some attempts to explore the analytical structure of the gluon propagator from numerical solutions of coupled DSEs of quarks, gluons and ghosts and from fits to lattice date, see Ref. [29] and references therein). In Refs. [31, 32], the authors proposed a confining, separable model gluon propagator with the rank-1 form

$$
g^{2} D_{\mu \nu}^{e f f}(p-q)=\delta_{\mu \nu} D_{0} f_{0}\left(p^{2}\right) f_{0}\left(q^{2}\right)
$$

where $f_{0}\left(p^{2}\right)=\exp \left(-p^{2} / \Lambda^{2}\right)$. This model is found to be successful in describing light flavor pseudo-scalar and vector meson observables with parameters $\Lambda_{0}=0.678 \mathrm{GeV}, D_{0} \Lambda_{0}^{2}=128.0$ and the degenerated light quark mass $m=6.6 \mathrm{MeV}$ [32].

In recent years, the rainbow-ladder DSE models were extended to finite temperature and chemical potential to investigate QCD thermodynamics (for a review, see [33]). As for the rank-1 confining separable model, the extension to finite temperature and chemical potential is accomplished by transcription of the Euclidean quark four-momentum via $q \longrightarrow$ $\widetilde{q}_{n}=\left(\vec{q}, \widetilde{\omega}_{n}\right)$, with $\widetilde{\omega}_{n}=\omega_{n}+i \mu$, and no new parameters are introduced [32, 34, 35]. This means the effective gluon propagator at finite temperature and chemical potential is modeled as

$$
g^{2} D_{\mu \nu}^{e f f}\left(\widetilde{p}_{k}-\widetilde{q}_{n}\right)=\delta_{\mu \nu} D_{0} f_{0}\left(\widetilde{p}_{k}^{2}\right) f_{0}\left(\widetilde{q}_{n}^{2}\right) .
$$

On the other hand, the finite temperature and chemical potential version of the rainbow-DSE of quark propagator (Eq. 15) reads

$$
G^{-1}\left(\widetilde{p}_{k}\right)=i \gamma \cdot \widetilde{p}_{k}+m+\frac{4}{3} T \sum_{n=-\infty}^{+\infty} \int \frac{d^{3} q}{(2 \pi)^{3}} g^{2} D_{\mu \nu}^{e f f}\left(\widetilde{p}_{k}-\widetilde{q}_{n}\right) \gamma_{\mu} G\left(\widetilde{q}_{n}\right) \gamma_{\nu}
$$


and the quark propagator is generally decomposed as

$$
G^{-1}\left(\widetilde{p}_{k}\right)=i \vec{\gamma} \cdot \vec{p} A\left(\widetilde{p}_{k}^{2}\right)+i \gamma_{4} \widetilde{\omega_{k}} C\left(\widetilde{p}_{k}^{2}\right)+B\left(\widetilde{p}_{k}^{2}\right)
$$

The rank-1 separable model leads to the rainbow-DSE solution: $A\left(\widetilde{p}_{k}^{2}\right)=C\left(\widetilde{p}_{k}^{2}\right)=1$ and $B\left(\widetilde{p}_{k}^{2}\right)=m+b(T, \mu) f_{0}\left(\widetilde{p}_{k}^{2}\right)$, where $b(T, \mu)$ satisfies the following equation

$$
b(T, \mu)=\frac{16}{3} D_{0} T \sum_{n=-\infty}^{+\infty} \int \frac{d^{3} q}{(2 \pi)^{3}} \frac{f_{0}\left(\widetilde{q}_{n}^{2}\right)\left[m+b(T, \mu) f_{0}\left(\widetilde{q}_{n}^{2}\right)\right]}{\left[\widetilde{q}_{n}^{2}+\left(m+b(T, \mu) f_{0}\left(\widetilde{q}_{n}^{2}\right)\right]^{2}\right.} .
$$

Of course, that the vector self-energy amplitudes $A$ and $C$ are unity does not cover all the physical effects, but this respects the nature of the separable model - the correspondence between quark propagator scalar self-energy amplitude $B$ and the separable gluon propagator form factor $f_{0}[35]$. Furthermore, it is an open question how the chemical potential $\mu$ enters the gluon propagator and the forgoing transcription of the fourth-component momentum $p_{4} \longrightarrow \widetilde{\omega}_{n}=\omega_{n}+i \mu$ has not been justified. However, this is again the requirement of the nature of the separable model and we think it worthwhile to allow such a uncomplicated extension to finite $T$ and $\mu$ in which the Matsubara modes are not coupled and one could sum them as easily as possible and then make a first survey of the thermodynamic observables [35]. Fortunately the results turn out to be satisfactory - the correspondence nature of the separable model plays a vital and correct role here [31].

Eq. (21) is numerically solved. Note that this simple model quite facilitates summation over fermion Matsubara frequencies as well as the 3-momentum integration due to its ultraviolet finiteness characterized by the Gaussian function $f_{0}\left(\widetilde{q}_{n}^{2}\right)$. As a result, no renormalization is needed. The imaginary part of $b(T, \mu)$ is found to be very small (of order $\sim 10^{-18}$ ) for all temperatures and chemical potentials while the real part takes strikingly different values at different $T$ and $\mu$ regions, hence serving as the order parameter. Fig. 1 shows the $\mu$-dependent behavior of $\operatorname{Re} b(T, \mu)$ at several fixed temperatures. One sees that at low temperatures, $\operatorname{Re} b(T, \mu)$ demonstrates an abrupt discontinuous decrease to a very small value at some critical chemical potential, which is typical of a phase transition of first order and resembles the observations of Nambu-Jona-Lasinio (NJL) model [36]. Nevertheless, at higher temperatures, $\operatorname{Re} b(T, \mu)$ shows a smooth, gradual decrease as $\mu$ increases, implying an analytic crossover. So one can naturally expect a CEP at which the first-order phase transition and the crossover connect. This will be verified by determining the most singular point of the quark number susceptibility, which is illustrated in subsection C. To summarize 
this subsection, we point out that the observations of Fig. 1 agree with the scenario of QCD phase transition shortly reviewed in the introduction.

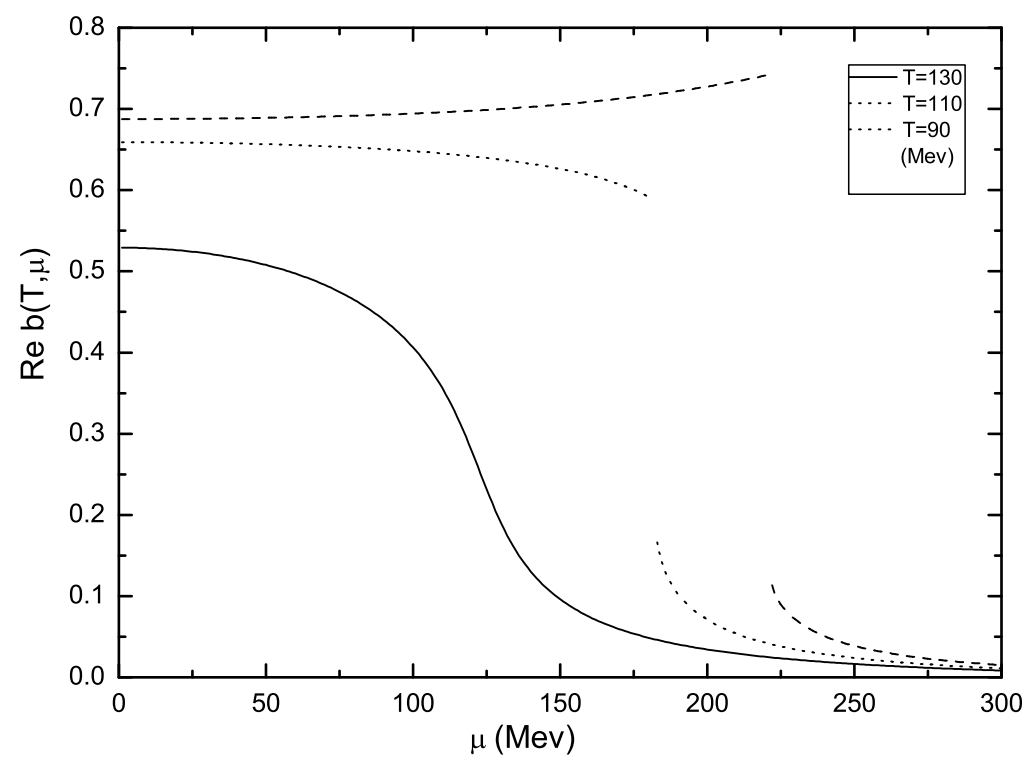

FIG. 1: $\mu$-dependence of $\operatorname{Re} b(T, \mu)$ at three fixed temperatures. The evolution from a first-order phase transition to a crossover is shown. The different monotonous behavior of $\operatorname{Re} b(T, \mu)$ in the symmetry broken phase for $T=90 \mathrm{MeV}$ and $T=110 \mathrm{MeV}$ may be ascribed to the artifact of the separable model.

\section{B. The dressed vector vertex}

We now turn to the dressed vector vertex. The finite temperature and chemical potential version of the inhomogeneous ladder BSE for the dressed vector vertex (the fourth component) Eq. (16) with vanishing total momentum reads

$$
\Gamma_{4}\left(\widetilde{p}_{k}, 0\right)=\gamma_{4}-\frac{4}{3} T \sum_{n=-\infty}^{+\infty} \int \frac{d^{3} q}{(2 \pi)^{3}} g^{2} D_{\rho \sigma}^{e f f}\left(\widetilde{p}_{k}-\widetilde{q}_{n}\right) \gamma_{\rho} G\left(\widetilde{q}_{n}\right) \Gamma_{4}\left(\widetilde{q}_{n}, 0\right) G\left(\widetilde{q}_{n}\right) \gamma_{\sigma}
$$

where the model gluon propagator $g^{2} D_{\rho \sigma}^{e f f}\left(\widetilde{p}_{k}-\widetilde{q}_{n}\right)$ is given by Eq. (18).

From Lorentz structure analysis, the dressed vector vertex with vanishing total momentum at $T=0=\mu$ can be decomposed as [30]

$$
\Gamma_{\mu}(p, 0)=\alpha_{1}\left(p^{2}\right) \gamma_{\mu}+\alpha_{2}\left(p^{2}\right) \gamma \cdot p p_{\mu}-\alpha_{3}\left(p^{2}\right) i p_{\mu}
$$


This can also be easily seen from the Ward identity $i \Gamma(p, 0)=\partial G^{-1}(p) / \partial p_{\mu}$ with $G^{-1}(p)=$ $i \gamma \cdot p A\left(p^{2}\right)+B\left(p^{2}\right)$. In Eq. (23), $\alpha_{1}\left(p^{2}\right)$ is the dominant term, while the other two terms contribute so little that they can even be neglected [30]. At finite temperature and chemical potential, we keep the corresponding "leading" term in $\Gamma_{4}\left(\widetilde{p}_{k}, 0\right)$ and add another term

$$
\Gamma_{4}\left(\widetilde{p}_{k}, 0\right)=\alpha\left(\widetilde{p}_{k}^{2}\right) \gamma_{4}-i \beta\left(\widetilde{p}_{k}^{2}\right) \widetilde{\omega}_{k}
$$

Here we stress that the second term is vital and indispensable for describing the QCD phase transition at finite temperature and chemical potential. Some explanation goes as follows in this connection. From the Ward identity Eq. (6) one sees that this term arises from the derivative of the scalar self-energy amplitude $B\left(\widetilde{p}_{k}^{2}\right)$ of the inverse dressed quark propagator Eq. (20) with respect to the chemical potential $\mu$. Note that $B\left(\widetilde{p}_{k}^{2}\right)$ experiences an abrupt decrease at some critical $\mu$ when a true phase transition occurs (on the contrary, the vector self-energy amplitudes $A\left(\widetilde{p}_{k}^{2}\right)$ and $C\left(\widetilde{p}_{k}^{2}\right)$ evolve smoothly with $T$ and $\mu$ [32]) and hence makes a considerable contribution to $\Gamma_{4}\left(\widetilde{p}_{k}, 0\right)$ at that point.

Putting the ansatz for the dressed vector vertex Eq. (24), the dressed quark propagator Eq. (20) and the confining separable model gluon propagator Eq. (18) into Eq. (22), one can transform the ladder BSE into two coupled non-linear equations

$$
\begin{array}{r}
u=\frac{8}{3} D_{0} T \sum_{l=-\infty}^{+\infty} \int \frac{d^{3} q}{(2 \pi)^{3}} f_{0}\left(\widetilde{q}_{l}^{2}\right) \frac{\left[1-u f_{0}\left(\widetilde{q}_{l}^{2}\right)\right]\left[\widetilde{\omega}_{l}^{2}-\vec{q}^{2}-\left(m+b f_{0}\left(\widetilde{q}_{l}^{2}\right)\right)^{2}\right]+2 v f_{0}\left(\widetilde{q}_{l}^{2}\right) \widetilde{\omega}_{l}\left[m+b f_{0}\left(\widetilde{q}_{l}^{2}\right)\right]_{25)}}{\left[\widetilde{q}_{l}^{2}+\left(m+b f_{0}\left(\widetilde{q}_{l}^{2}\right)\right)^{2}\right]^{2}} \\
v=\frac{16}{3} D_{0} T \sum_{l=-\infty}^{+\infty} \int \frac{d^{3} q}{(2 \pi)^{3}} f_{0}\left(\widetilde{q}_{l}^{2}\right) \frac{v f_{0}\left(\widetilde{q}_{l}^{2}\right)\left[\widetilde{q}_{l}^{2}-\left(m+b f_{0}\left(\widetilde{q}_{l}^{2}\right)\right)^{2}\right]-2\left[1-u f_{0}\left(\widetilde{q}_{l}^{2}\right)\right] \widetilde{\omega}_{l}\left[m+b f_{0}\left(\widetilde{q}_{l}^{2}\right)\right]_{26)}}{\left[\widetilde{q}_{l}^{2}+\left(m+b f_{0}\left(\widetilde{q}_{l}^{2}\right)\right)^{2}\right]^{2}}
\end{array}
$$

with $\alpha\left(\widetilde{p}_{k}^{2}\right)=1-u(T, \mu) f_{0}\left(\widetilde{p}_{k}^{2}\right)$ and $\beta\left(\widetilde{p}_{k}^{2}\right) \widetilde{\omega}_{k}=v(T, \mu) f_{0}\left(\widetilde{p}_{k}^{2}\right)$. With $b(T, \mu)$ obtained from Eq. (21), these two equations are numerically solved. Here, one also notes that the Gaussian function $f_{0}\left(\widetilde{q}_{l}^{2}\right)$ appearing in the numerators of the integrands justifies a numerical cut-off for summation over Matsubara frequencies as well as 3-D momentum integration.

\section{Results for the quark number susceptibility}

Having completed the calculation of the dressed quark propagator and the dressed vector vertex, we are now in a position to calculate the quark number susceptibility. The numerical results are shown in Fig. 2, where $\chi(T, \mu)$ is normalized by the free quark quark number susceptibility in the chiral limit $\chi^{\text {free }}(T, \mu)$ (Eq. (14)) and hence dimensionless. 


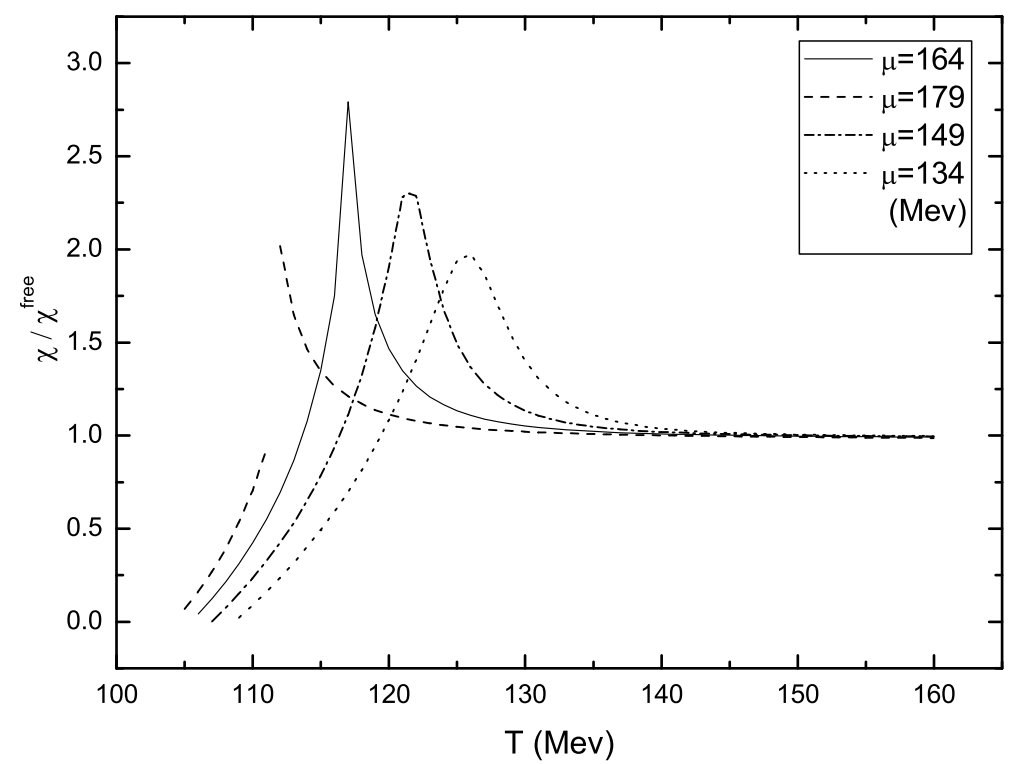

FIG. 2: $T$-dependence of $\chi(T, \mu)$ at four fixed chemical potentials. From left to right, $\mu=$ $179,164,149,134 \mathrm{MeV}$, respectively. The CEP is identified as the diverging cusp developed in the solid line at $T_{\mathrm{CEP}}=117 \mathrm{MeV}, \mu_{\mathrm{CEP}}=164 \mathrm{MeV}$.

Let us analyse the observations from Fig. 2. The most singular point develops along the $\mu_{\mathrm{CEP}}=164 \mathrm{MeV}$ solid line at $T_{\mathrm{CEP}}=117 \mathrm{MeV}$. This is the CEP, at which the quark number susceptibility manifests itself as a diverging cusp that should translate into event by event fluctuations of baryon multiplicity [22]. For larger $\mu=179 \mathrm{MeV}$ (the dashed line), $\chi(T, \mu)$ also jumps across the chiral phase transition and has a discontinuity due to its firstorder character. The peak is still pronounced but its height much suppressed. For smaller chemical potentials ( $\mu=149,134 \mathrm{MeV}$, the dash-dotted and the dotted line), one enters a crossover region, in which the discontinuity of $\chi(T, \mu)$ at the transition vanishes and $\chi(T, \mu)$ evolves gradually with $T$ in a smoother and smoother manner.

Another observation from Fig. 2 is that far below the chiral phase transition, $\chi(T, \mu)$ is greatly suppressed and at asymptotic temperatures, $\chi(T, \mu)$ tends unambiguously to the value of susceptibility of a free quark gas. In the latter respect, our demonstration is more akin to that of lattice predictions [23, 25] than NJL-type models [18, 20], where $\chi$ decreases too fast at asymptotic temperatures or chemical potentials. This may be accounted for by the fact that the momentum-dependent interaction DSE model represents a better asymptotic 
freedom behavior.

Our calculation determines the CEP location at $T_{\mathrm{CEP}}=117 \mathrm{MeV}$ and $\mu_{\mathrm{CEP}}=164 \mathrm{MeV}$. The most recent experimental estimate by extracting $\eta / s$ as a function of $T$ and $\mu$ from an elliptic flow excitation function gives $T_{\mathrm{CEP}} \sim 165-170 \mathrm{MeV}$ and $\mu_{\mathrm{CEP}} \sim 150-180 \mathrm{MeV}$ [16], which is in good agreement with lattice prediction with almost realistic pion mass [24]. So very encouragingly, our results for $\mu_{\mathrm{CEP}}$ lies exactly in this interval, whereas our $T_{\mathrm{CEP}}$ deviates a lot, for the pseudo-critical temperature $T_{c}$ in our calculation takes a relatively lower value (see below). As for NJL-type models, they predict a much higher $\mu_{\mathrm{CEP}}$ [18, 20, 22]. In fact, NJL-type models have a common tendency to lead to the CEP at relatively high $\mu_{\mathrm{CEP}} \sim 300 \mathrm{MeV}[22]$.

Having identified the CEP, we now show the complete phase diagram obtained from our model, which assumes the shape sketched in Fig. 3. Two separate phases are shown in

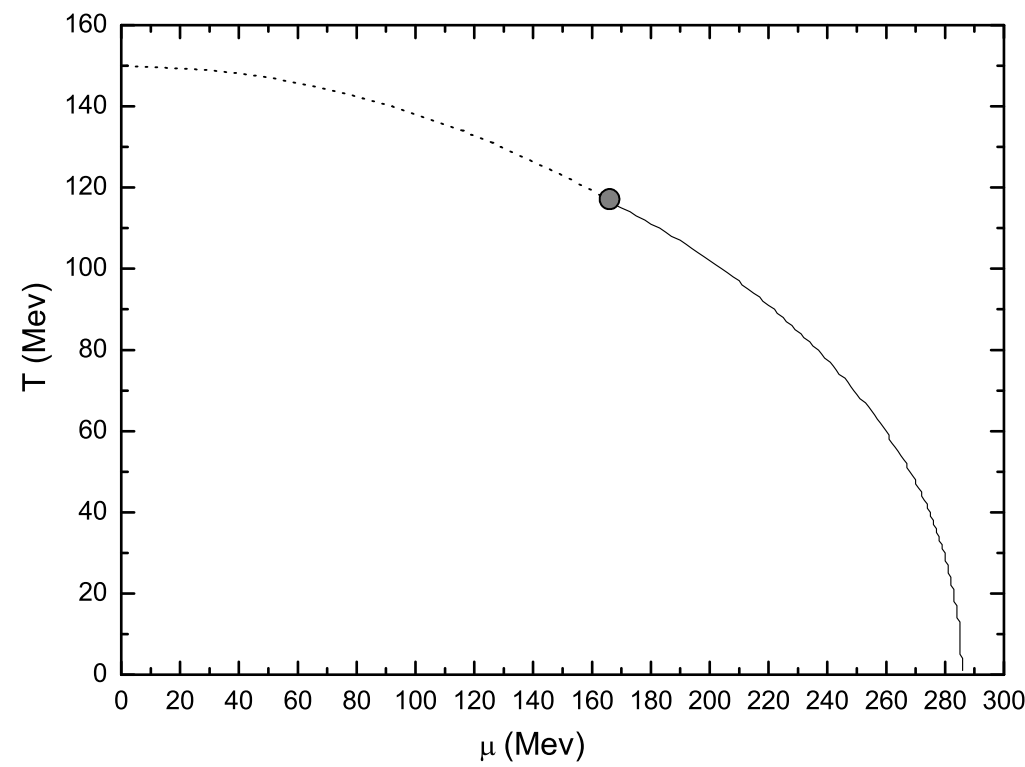

FIG. 3: The phase diagram characterized by $\operatorname{Re} b(T, \mu)$. The solid line represents the first-order phase transition and the dotted line the crossover transition. The filled circle indicates the CEP.

the $T-\mu$ plane: the chiral symmetry restored phase and the phase with (approximate) chiral symmetry dynamically broken. The solid line represents a first-order phase transition line, which begins from $\mu_{c}=286 \mathrm{MeV}$ at vanishing temperature and ends at the CEP $\left(T_{\mathrm{CEP}}=117 \mathrm{MeV}, \mu_{\mathrm{CEP}}=164 \mathrm{MeV}\right)$. Beyond the $T_{\mathrm{CEP}}$, one ends up with a crossover 
(the dotted line) with a pseudo-critical temperature $T_{c}=150 \mathrm{MeV}$ at vanishing chemical potential.

Last but not least, to check the parameter sensitivity of the location of the critical end point, we first vary the degenerated current quark mass $m$, since the value of $m$ is believed to be closely associated with the nature of the phase transition. The numerical results is shown in Fig. 4.

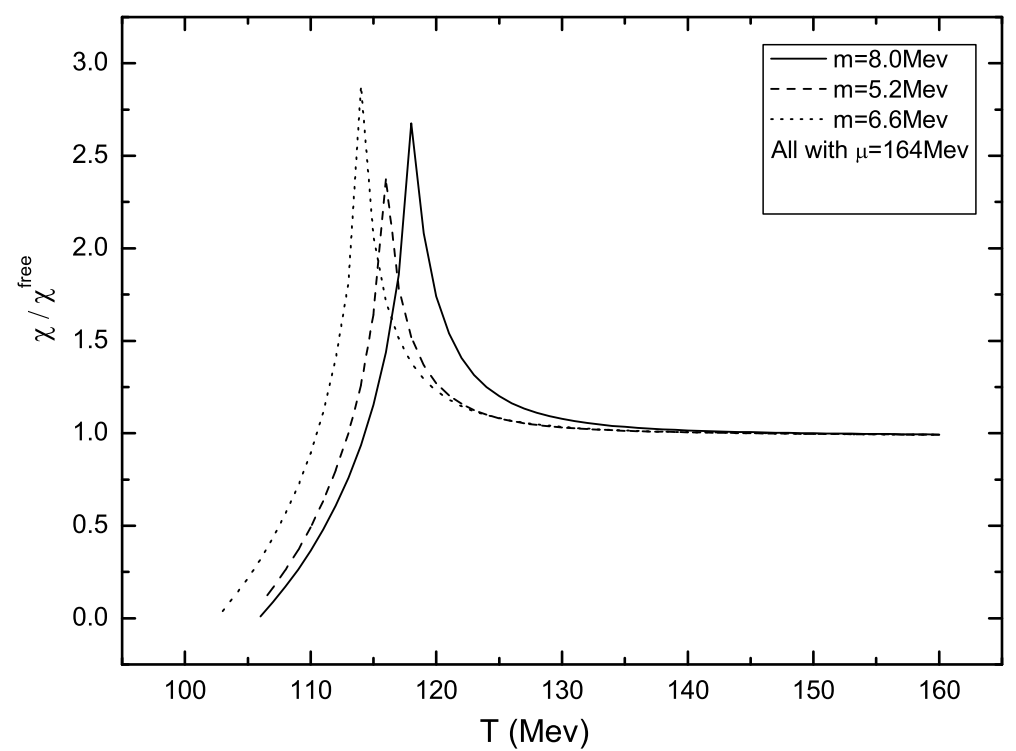

FIG. 4: The location of the critical end point for $m=5.2 \mathrm{MeV}$ and $m=8.0 \mathrm{MeV}$ with "physical" $D_{0}=128.0 /(0.687 \mathrm{GeV})^{2}$ and for "physical" $m=6.6 \mathrm{MeV}$ with $D_{0}=128.0 /(0.700 \mathrm{GeV})^{2}$. For all three curves, the chemical potential takes the same value $\mu_{\mathrm{CEP}}=164 \mathrm{MeV}$.

We find that when the quark mass value varies from $m=5.2 \mathrm{MeV}$ to $m=8.0 \mathrm{MeV}$, the most singular point of the quark number susceptibility emerges always along the $\mu_{\mathrm{CEP}}=$ $164 \mathrm{MeV}$ line, while the corresponding $T_{\mathrm{CEP}}$ has a very minor shift from $116 \mathrm{MeV}$ to $118 \mathrm{MeV}$ (the dashed and solid line, respectively). As we change the coupling parameter from its physical value in the present model $D_{0}=128.0 /(0.687 \mathrm{GeV})^{2}$ to $D_{0}=128.0 /(0.700 \mathrm{GeV})^{2}$ (with quark mass fixed at its physical value), the corresponding CEP moves slightly to $T_{\mathrm{CEP}}=114 \mathrm{MeV}$, with its $\mu_{\mathrm{CEP}}$ remaining at $164 \mathrm{MeV}$. Lastly, as for the parameter $\Lambda$, the effect of its variation can be canceled by taking a larger numerical cutoff in practical calculations, since it appears in the exponential as the denominator. Also notable here is that the critical line does not change much, either for $m=5.2 \mathrm{MeV}$, the critical chemical 
potential at vanishing temperature is $\mu_{c}=280 \mathrm{MeV}$, for $m=8.0 \mathrm{MeV}, \mu_{c}=289 \mathrm{MeV}$, and for the new coupling parameter $D_{0}=128.0 /(0.700 \mathrm{GeV})^{2}, \mu_{c}=281 \mathrm{MeV}$, while all the pseudo-critical temperatures take almost the same value as that for the "physical" parameters.

The conclusion is that the location of the CEP (especially $\mu_{\mathrm{CEP}}$ ) and the critical line is relatively robust with respect to the variations of the model parameters around their physical values.

\section{SUMMARY AND CONCLUSIONS}

The primary goal of this paper is to identify and locate the CEP through a continuum study of the quark number susceptibility. To this end, we first derive a model-independent integral formula expressing the quark number susceptibility in terms of dressed quark propagator and dressed vector vertex. The latter objects are then confronted in the rainbow-ladder DSE-BSE framework with a confining separable model gluon propagator. With these results, the quark number susceptibility is calculated. The quark number susceptibility measures the response of the quark number density to changes in the baryon chemical potential and is of particular interest for exploring the CEP in that it is expected to diverge at the CEP. Our calculation reproduces this diverging phenomenon, which may be detectable in heavy ion collision experiments [10, 25].

The location of the CEP our calculation predicts lies at a reasonable value. Interestingly the $\mu_{\mathrm{CEP}}$ is coincident with recent experimental estimate and more acceptable than NJL-type model predictions. In this regard, we emphasize that the confining separable DSE model admits a momentum-dependent effective interaction, which is not the case for NJL-type models. Moreover, the rainbow-ladder truncation scheme for DSE-BSE respects the axialvector Ward-Takahashi identity (AV-WTI) [30], which relates closely to the chiral symmetry and its dynamical breaking, and the usual Abelian WTI, which plays an essential part in our analysis in this paper.

Nevertheless, our model study says nothing about the recently prevalent terminology in QCD thermodynamics, namely the strongly coupled Quark-Gluon Plasma(sQGP) [37]. $\eta / s$, rather than the quark number susceptibility, is believed to provide a good characteristic of sQGP liquid and its surprisingly small value makes a current focus [15, 37], with which we 
are also concerned.

\section{ACKNOWLEDGMENT}

We acknowledge helpful communications with P. C. Tandy and D. Blaschke on the confining separable model. This work was supported in part by the National Natural Science Foundation of China (under Grant Nos 10575050 and 10775069) and the Research Fund for the Doctoral Program of Higher Education (under Grant No 20060284020).

[1] M. Stephanov, arXiv:hep-lat/0701002 v1 (2006).

[2] U. Heinz, arXiv:hep-ph/0407360v1 (2004).

[3] M. He, D. K. He, H. T. Feng, W. M. Sun, and H. S. Zong, Phys. Rev. D 76, 076005 (2007).

[4] F. Wilczek, Nature 443, 637 (2006).

[5] Y. Aoki et al., Nature 443, 675 (2006).

[6] Y. Aoki et al., Phys. Lett. B 643, 46 (2006).

[7] F. Karsch, arXiv:hep-lat/0711.0656v1 (2007).

[8] O. Philipsen, arXiv:hep-lat/0708.1293v2 (2007).

[9] M. Stephanov, Prog. Theor. Phys. Suppl. 153, 139(2004); Int. J. Mod. Phys. A 20, 4387 (2005).

[10] M. Stephanov, K. Rajagopal, and E. Shuryak, Phys. Rev. Lett. 81, 4816 (1998); Phys. Rev. D 60, 114028 (1999).

[11] Y. Hatta and M. A. Stephanov, Phys. Rev. Lett. 91, 102003 (2003).

[12] Z. Fodor and S. D. Katz, JHEP 04, 050 (2004); Shinji Ejiri, Phys. Rev. D 77, 014508 (2008).

[13] M. A. Stephanov, Phys. Rev. D 73, 094508 (2006).

[14] B. Lungwitz and M. Bleicher, Phys. Rev. C 76, 044904 (2007).

[15] R. A. Lacey et al., Phys. Rev. Lett. 98, 092301 (2007).

[16] R. A. Lacey et al., arXiv:nucl-ex/0708.3512v6 (2008).

[17] Yoshitaka Hatta and Takashi Ikeda, Phys. Rev. D 67, 014028 (2003).

[18] P. Costa, C. A. de Sousa, M. C. Ruivo and Yu. L. Kalinovsky, Phys. Lett. B 647, 431 (2007);

P. Costa, M. C. Ruivo, C. A. de Sousa, Phys. Rev. D 77, 096001 (2008). 
[19] C. Sasaki, B. Friman, and K. Redlich, Phys. Rev. D 75, 074013 (2007).

[20] B.-J. Schaefer and J. Wambach, Phys. Rev. D 75, 085015 (2007).

[21] K. Redlich, B. Friman, and C. Sasaki, J. Phys. G: Nucl. Part. Phys. 35044013 (2008).

[22] Kenji Fukushima, Phys. Rev. D 77, 114028 (2008).

[23] C. R. Allton et al., Phys. Rev. D 71, 054508 (2005).

[24] R.V. Gavai and Sourendu Gupta, Phys. Rev. D 71, 114014 (2005).

[25] C. Schmidt, arXiv:hep-lat/0610116v2 (2006); O. Philipsen, arXiv:hep-lat/0510077 (2005).

[26] L. P. Csernai, J. I. Kapusta, and L. D. McLerran, Phys. Rev. Lett. 97, 152303 (2006); J.-W. Chen and E. Nakano, arXiv:hep-ph/0604138 (2006).

[27] C. D. Roberts, and A. G. Williams, Prog. Part. Nucl. Phys. 33, 477 (1994).

[28] R. Alkofer and L. von Smekal, Phys. Rep. 353, 281 (2001).

[29] C. S. Fischer, J. Phys. G 32, R253 (2006).

[30] P. Maris and C. D. Roberts, Int. J. Mod. Phys. E 12, 297 (2003); A. Holl, C. D. Roberts, and S. V. Wright, nuch-th/0601071 v1 (2006).

[31] C. J. Burden, L. Qian, C. D. Roberts, P. C. Tandy, and M. J. Thomson, Phys. Rev. C 55, 2649 (1997).

[32] D. Blaschke et al., Int. J. Mod. Phys. A 16, 2267 (2001).

[33] C. D. Roberts and S. M. Schmidt, Prog. Part. Nucl. Phys. 45, S1 (2000).

[34] D. Blaschke and P.C. Tandy, arXiv:nucl-th/9905067v1 (1999); D.Blaschke and C. D. Roberts, Nucl. Phys. A 642, 197 (1998).

[35] Private communications with P. C. Tandy and D. Blaschke.

[36] M. Buballa, Phys. Rep. 407, 205 (2005).

[37] E. Shuryak, Nucl. Phys. A 774, 387 (2006); arXiv:hep-ph/0804.1373v1 (2008). 